cofactor, NADPH, can then transport electrons to the next enzyme in a cascade (Fig. 1a). ITO electrodes provide a high localized concentration of electrons, and Megarity et al. anticipated that they could augment the natural FNR system by replacing ferredoxin with a nanoporous ITO surface that would co-entrap FNR with a partner enzyme that performs the final step in a cascade (Fig. 1b).

To demonstrate this concept, the authors first established that FNR and a second enzyme (alcohol dehydrogenase; ADH) can be co-entrapped in the disordered pores of ITO electrodes. When both FNR and ADH were adsorbed onto the electrode and placed in a solution containing $\mathrm{NADP}^{+}$and a substrate for $\mathrm{ADH}$, the authors observed the formation of a reaction product. They also observed an electric current, the size of which depended on the amount of FNR present, indicating that FNR-mediated electron shuttling was occurring.

Megarity et al. found that the enzymeloaded electrode could be reused by rinsing it thoroughly with water and then plunging it into a fresh solution of the reaction substrate. This not only demonstrated the robustness of their set-up, but also showed that both FNR and $\mathrm{ADH}$ become tightly bound within the electrode pores. By contrast, the authors found that $\mathrm{NADP}^{+}$is mostly not retained in the pores, and must be added to the reaction solution to restore a high reaction rate.

The authors' system simplifies electron transport and accelerates the rate of cofactor regeneration for cascades initiated by the transfer of electrons to FNR. The researchers estimate that this nanoconfinement strategy leads to a local concentration of 1.6 millimolar - about 1,000 times higher than solution concentrations - for each catalytic component in a pore, which reduces the distance required for the cofactor to travel between FNR and ADH compared with the distance required if the reaction is performed purely in solution. As a result, the overall rate of product formation is increased. Megarity and colleagues calculate that each 'minimal catalytic unit' in the reaction - the smallest number of enzyme molecules and cofactors needed for a reaction to occur; in this case, one FNR, one ADH and one NADPH - can produce about 125 molecules of product each second, which is a feat for a cascade involving a series of electron transfers.

Encouragingly, the authors show that three other NADPH-dependent enzymes can be used in place of ADH in their system to catalyse a variety of reduction reactions. This indicates that the nanoconfinement approach could have broad utility for NADPH-dependent biocatalytic transformations - although the observed reaction kinetics for each enzyme were different. Further work is required to define the full scope of NADPH-dependent enzymes and classes of enzyme powered by non-NADPH cofactors that are compatible with this strategy.

For example, testing reductase enzymes other than FNR in the authors' system will reveal whether porous electrodes can generally act as a source of electrons for proteins in electron-shuttling pathways, and whether this strategy can be used to recycle cofactors other than NADPH. Many classes of enzyme rely on electron transport, and a general strategy that would allow them to be used effectively in reactions outside biological systems could dramatically improve the scalability of such reactions. It could also facilitate the study of enzymes for which the electron-supplying partners are unknown.

Megarity and colleagues' work explores the nanoconfinement of two enzymes. One could also imagine packing additional enzymes into electrode pores to increase the efficiency of more-complex multi-enzyme cascades. The use of a synthetic compartmentalization system could thus find application in the enzymatic production of both the simple 'commodity' chemicals produced at large scales for the chemical industry, and structurally complex molecules, such as pharmaceutical agents.

Alison Narayan is in the Department of Chemistry, Life Sciences Institute, University of Michigan, Ann Arbor, Michigan 48109, USA.

e-mail:arhardin@umich.edu

1. Megarity, C. F. et al. Angew. Chem. Int. Edn https:// doi.org/10.1002/anie.201814370 (2019).

2. Sheldon, R. A. \& Woodley, J. M. Chem. Rev. 118, 801-838 (2018).

3. Ricca, E., Brucher, B. \& Schrittwieser, J. H. Adv. Synth. Catal. 353, 2239-2262 (2011).

4. Li, Y. \& Cirino, P. C. Biotechnol. Bioeng. 111 1273-1287 (2014).

5. Roy, A. et al. J. Am. Chem. Soc. 136, 17343-17349 (2014).

6. Munro, A. W. \& McLean, K. J. in Encyclopedia of Biophysics (ed. Roberts, G. C. K.) 601-605 (Springer, 2013).

7. Cardoso, A. R. et al. Free Radical Biol. Med. 52, 2201-2208 (2012)

8. Avalos, J. L., Fink, G. R. \& Stephanopoulos, G. Nature Biotechnol. 31, 335-341 (2013).

9. Siritanaratkul, B. et al. Chem. Sci. 8, 4579-4586 (2017).

This article was published online on 8 March 2019.

\title{
ASTRONOMY
}

\section{X-ray chimneys in the Galactic Centre}

\section{X-ray observations of the Galactic Centre have uncovered chimney-like structures filled with hot plasma. The discovery might reveal how energy is transported from this central region to far-off locations. SEE LETTER P.347}

\section{MASHA CHERNYAKOVA}

$\mathrm{T}$ The centre of our Galaxy hosts a supermassive black hole that currently emits electromagnetic radiation extremely weakly, but could have been much more active in the past. Observations of $\gamma$-rays have revealed two huge structures known as Fermi bubbles located above and below the Galactic plane ${ }^{1}$. These bubbles are filled with highly energetic particles moving at close to the speed of light, which were released from the Galactic Centre a few million years ago. On page 347, Ponti et al. ${ }^{2}$ report X-ray observations that reveal chimney-like structures connecting the region around the Galactic Centre to the Fermi bubbles.

The authors used more than 750 hours of $\mathrm{X}$-ray observations made by the space-based XMM-Newton and Chandra telescopes to obtain the first detailed X-ray map of the central region of our Galaxy, an area of around $300 \times 500$ parsecs. (For comparison, the distance from Earth to the Galactic Centre is about 8,000 parsecs.) This map reveals two elongated, quasi-linear structures, each about 160 parsecs in length, above and below the supermassive black hole at the centre of the Milky Way (Fig. 1). Ponti et al. name these two structures the northern and southern Galactic Centre chimneys.

Previous X-ray and radio observations revealed two smaller lobes of outflowing matter, at a scale of about 15 parsecs, located above and below the Galactic plane $e^{3,4}$. The chimney structures connect these lobes to the Fermi bubbles, which start from about 100 parsecs above the Galactic plane and occupy a huge region approaching the size of the Galaxy itself.

The similarities between the northern and southern chimneys suggest that they have a common origin, most probably connected to the Galactic Centre. The chimneys seem to be well confined in the direction along the Galactic plane and have sharp edges at their vertical extents. Both are filled with a hot plasma (at a temperature of about 8 million kelvin) and have a total luminosity about a million times greater than that of the Sun.

The observed temperature and luminosity are consistent with the idea that the plasma in the chimneys is heated by energy released during the explosion of massive stars concentrated 


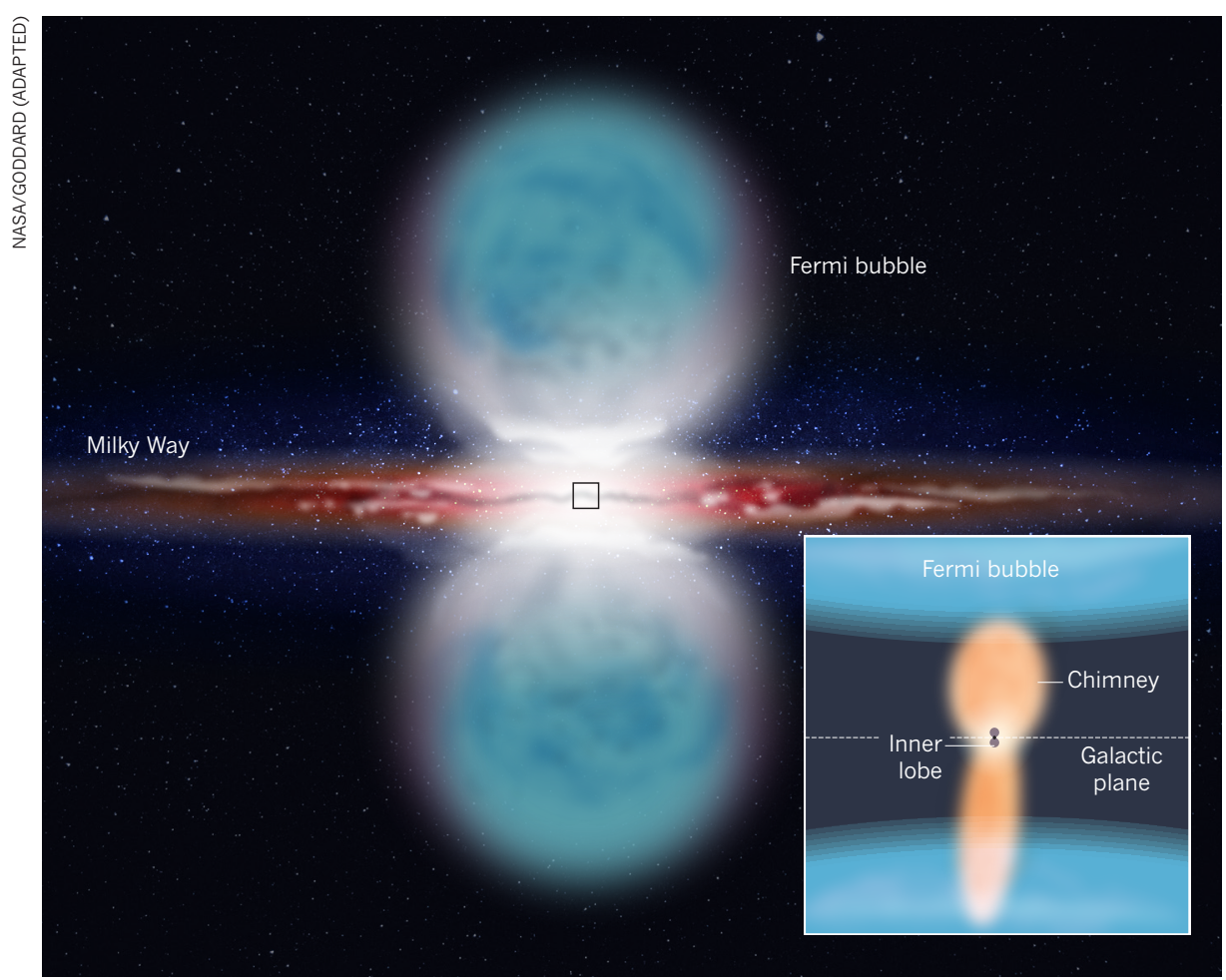

Figure 1 | Galactic chimneys and Fermi bubbles. Two huge structures, known as Fermi bubbles, are located above and below the plane of our Galaxy ${ }^{1}$, and are centred on the supermassive black hole at the Galactic Centre. The bubbles are filled with highly energetic particles released from the Galactic Centre a few million years ago. X-ray and radio observations have also shown a much smaller pair of lobes of outflowing matter coming from the Galactic Centre. Ponti et al. ${ }^{2}$ now report X-ray observations that reveal two 'chimney' structures that seem to connect the inner lobes to the Fermi bubbles.

near the chimney bases. However, although the plasma filling the smaller, inner lobes is also heated by supernovae, the new X-ray data do not support the idea that the chimneys are a direct continuation of the inner lobes. It is more likely that massive stars in the Galactic Centre are distributed non-uniformly, leading to the formation of separate outflows: a small one close to the supermassive black hole, and larger ones at the chimney bases.

The morphology of the chimneys suggests that they could be channels excavated by the powerful outflows from the supernovae at the chimney bases. Ponti and colleagues suggest that the chimneys might transport energy from the active region of the Galactic Centre to the Fermi bubbles. Various models have been used to estimate the power needed to create and sustain the Fermi bubbles, but the estimates differ by several orders of magnitude $e^{1,5,6}$. The lower end of these estimates comes from models in which the Fermi bubbles were created by a population of cosmic rays (consisting of protons and heavier ions) produced during supernova explosions in the Galactic Centre $^{5,7}$. The energy involved in this scenario is in the same ballpark as the observed energetics of the chimneys. It should be noted that the observed energy values are at the lower limit of the total energy produced by the chimneys, because only a fraction of the total energy will be deposited as X-ray-emitting plasma.

In this supernova-powered scenario, the supermassive black hole has only a secondary role. However, the morphology of the central Galactic region could also be the result of extremely energetic outbursts produced after the accumulation of gravitationally disrupted stars close to the black hole ${ }^{8}$. In this case, the chimneys could facilitate the propagation of matter and energy from the black hole to rarefied, low-pressure regions above the Galactic $\operatorname{disk}^{7}$. The black hole might also generate outflows that are thousands of times more energetic than its observed radiation, as is the case for some other galaxies ${ }^{9}$.

The discovery of the chimneys is another jigsaw piece in our picture of the complex processes that govern our Galaxy. But there are still many questions to be answered. At present, only part of the region at the base of the Fermi bubbles has been observed. Observations of the whole of this region will help to clarify whether the X-ray emissions from the chimneys are traces of outflows of gas passing through the inner lobes, or come from the heated gas surrounding the lobes. Detailed radio observations of the region occupied by the chimneys, and comparison of the features revealed by radio and X-ray observations, are also needed to help us better understand the energy transport from the Galactic Centre to Fermi bubbles.

Masha Chernyakova is at the School of Physical Sciences and at the Centre for

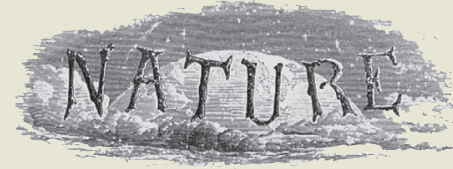

50 Years Ago

Conservationists, farmers and highway managers met in London on March 14 to discuss the function and management of road verges ... all that is needed is cooperation for roadside verges to become a haven of rare and attractive plants while not interfering with the safety and amenity of what is described under Common Law as "a perpetual right of passage for the Queen and all her subjects" ... [V] erges represent the last vestige of grasslands which existed before the modernization of agriculture. The rich pastures of the past often survive only on unploughed and unsprayed verges. Many roadside verges also provide a habitat for some of Britain's rare plants ... at least twenty-seven of the three hundred rarest species grow on roadside verges; Linum anglicum, perennial flax, grows only in this habitat and the same goes for several other species. Clearly the loss of one roadside verge could mean the end of a species in Britain. From Nature 22 March 1969

\section{Years Ago}

The Times of March 17 gives an account ... of a remarkable Australian rainfall. It states that "the extraordinary rainfall at Melbourne threatens the greatest flood since 1891. The south-eastern corner of Victoria and New South Wales is almost engulfed ... At Macedon 8 in. were registered in twenty-four hours, and other watersheds have been converted into lakes. Thousands of persons are homeless. Thirteen inches of rain in twenty-four hours has practically drowned the township of East Bellingen, in New South Wales. Although the damages are estimated to aggregate tens of thousands of pounds, the benefits from the breaking of the drought will be represented by hundreds of thousands."

From Nature 20 March 1919 
Relativity and Astrophysics (CfAR), Dublin City University, Glasnevin, Dublin 9, Ireland. e-mail:masha.chernyakova@dcu.ie

1. Su, M., Slatyer, T. R. \& Finkbeiner, D. P. Astrophys. J. 724, 1044-1082 (2010).

2. Ponti, G. et al. Nature 567, 347-350 (2019).
3. Morris, M. et al. Astron. Nachr. 324 (suppl.), 167-172 (2003)

4. Zhao, J.-H., Morris, M. R. \& Goss, W. M. Astrophys. J. 817, 171-187 (2016)

5. Crocker, R. M., Aharonian, F. Phys. Rev. Lett. 106 , 101102 (2011).

6. Kataoka, J. et al. Galaxies 6, 27 (2018).
7. Lacki, B. C. Mon. Not. R. Astron. Soc. 444, L39-L43 (2014).

8. Roberts, S. R., Jiang, Y.-F., Wang, Q. D. \& Ostriker, J. P. Mon. Not. R. Astron. Soc. 466, 1477-1490 (2017).

9. Churazov, E., Forman, W., Jones, C. \& Böhringer, H. Astron. Astrophys. 356, 788-794 (2000).

\section{The ripple effect of a single neuron}

The contribution of a single neuron to brain function might seem negligible. But a map of the influence of single neurons reveals a complex pattern that prevents redundancy and enables clear messaging. SEE ARTICLE P.334

IKUKO T. SMITH

$\mathrm{W}$

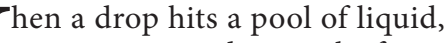
concentric cascading ripples form. Studying these ripples gives us information about the properties of the liquid and its molecules, such as their identity, mass, density or velocity. On page 334, Chettih and Harvey $^{1}$ take a similar approach to unravel the functional properties of the neural circuitry of the primary visual cortex in mouse brains: they trigger a small increase in the activity of a single neuron that causes a ripple of network activity in a pool of neurons.

The authors used a method that they call influence mapping to directly measure the effect of individual neurons on the activity of neighbouring ones. They genetically modified mice so that light-sensitive ion channels were expressed in neurons, and then used light to control neural activity (an approach called optogenetics). The mice were also made to express a fluorescent indicator of calcium concentration, which works as a reporter of the neurons' electrical activity (a technique called calcium imaging). Optogenetic stimulation

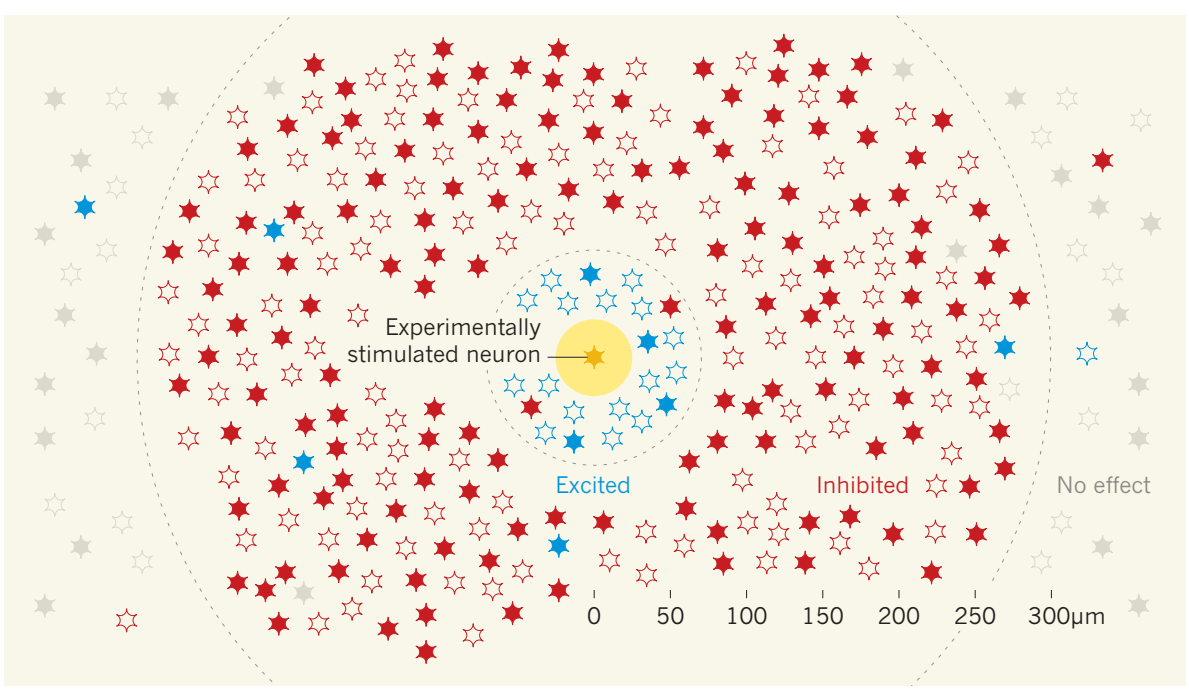

Figure 1 | Influence of a single neuron on the surrounding network. Chettih and Harvey ${ }^{1}$ describe a model of network computation in the mouse visual cortex. They found that experimental stimulation of a single neuron (yellow) predominantly caused widespread inhibition (red) of the activity of neighbouring neurons. Stimulation also had an excitatory effect (blue) at short distances $(25-70 \mu \mathrm{m})$, which affected a small proportion of neighbouring neurons, and almost no effect (grey) on neurons at long distances (more than $300 \mu \mathrm{m}$ ). Full stars represent neurons tuned to respond to similar features of the visual stimuli to the target neuron, and outlined stars represent neurons tuned differently. Within the area of predominant inhibition of neural activity $(70-300 \mu \mathrm{m})$, neurons similarly tuned to the target neuron were more strongly inhibited than were differently tuned ones. This general like-to-like suppression regime (called feature competition) was peppered with sparsely distributed nodes of activating influence involving a small number of neurons whose visual responses were highly similar to that of the target neuron (feature amplification). Together, these two modes of information processing minimize redundancy and promote accurate coding of visual stimuli. triggered several spikes of activity (action potentials) in target neurons. Chettih and Harvey then used calcium imaging to measure the influence of a single manipulated neuron on the spiking activity of neighbouring neurons.

A few extra action potentials in a single neuron are but a drop in the bucket for a complex, intertwining network of neurons. But when the authors considered the distance between the target and neighbouring neurons, as well as their functional characteristics (that is, whether they respond in similar or different ways to visual stimuli), a meaningful picture emerged. Their model of functional connectivity gives us a glimpse of the fundamental computation performed in the primary visual cortex.

In the past 15 years, studies of single neurons in live animals have improved our knowledge of how individual neurons are connected in, and contribute to the overall function of, their network ${ }^{2-7}$. Many of these studies have focused on the neural circuitry of the cortex, in which the connections between neurons, called synapses, are abundant but generally weak ${ }^{5}$. These properties of cortical synapses suggest a type of network computation in which the spiking activity of a single neuron would mean very little. Yet experimental evidence has shown that stimulation of a single neuron can have behavioural consequences.

For example, electrical stimulation of a single neuron in the motor cortex can cause whisker movements in mice ${ }^{6}$. In rats, a similar stimulation of individual neurons in the barrel cortex, which is involved in processing sensory information, can trigger a licking behaviour associated with touch perception ${ }^{7}$. Curiously, cortical networks seem to be sensitive to the activation of some, but not all, stimulated neurons $^{6,7}$. Chettih and Harvey provide an explanation for this observation.

The influence-mapping approach makes it possible to analyse a larger population of neurons than those studied using electrophysiology $y^{4,8}$. More importantly, it enabled the authors to categorize neuronal influences according to the degree of similarity between the responses of the manipulated neuron and those of the neurons it affects ${ }^{2,3}$. Notably, the subtle stimulation of neurons achieved with optogenetics is unlikely to elicit a strong generalized response and change the brain's state ${ }^{9}$. This approach might more closely mirror the basic computation of the cortical circuitry than other approaches.

Chettih and Harvey predominantly observed widespread suppression of neural activity around the target neuron (Fig. 1). 